\title{
INDEPENDENT FAMILIES IN COMPLETE BOOLEAN ALGEBRAS
}

BY

\author{
B. BALCAR AND F. FRANEK
}

\begin{abstract}
We present a proof (without any set-theoretical assumptions) that every infinite complete Boolean algebra includes a free subalgebra of the same cardinality. It follows that the set of all ultrafilters on an infinite complete Boolean algebra $B$ has power $2^{|B|}$.
\end{abstract}

1. Problems and results. Let $B$ be a Boolean algebra and $\operatorname{St}(B)$ its Stone space, i.e. the set of all ultrafilters on $B$ with the usual topology. If $B$ is finite, then obviously $|\operatorname{St}(B)|=\log _{2}|B|,{ }^{1}$ see [Ma]. The classical result due to Hausdorff [H] and Pospišil [P1] states that for a power set Boolean algebra $B=\mathscr{P}(\kappa)$ for any infinite cardinal $\kappa$, $|\operatorname{St}(B)|=2^{2^{\kappa}}=2^{|B|}$. The key role in their proof is played by the notion of an independent family of sets and the fact that there is such a family of subsets of $\kappa$ of size $2^{\kappa}$. The very natural problem of whether this can be generalized for all infinite complete Boolean algebras was first formulated (as far as we know) by Efimov [E]. Partial positive solutions were known from works of Kesl'yakov [K], Koppelberg [Ko], Monk [M] and Blaszczyk [Bla]. A short historical survey of this problem can be found in [Bla]. Independent systems in complete Boolean algebras are studied in Vladimirov's book.

We shall present proofs (in ZFC only) of the following theorems.

THEOREM A. In every infinite complete Boolean algebra $B$ there is an independent family $\mathscr{F} \subseteq B$ so that $|\mathscr{F}|=|B|$.

THEOREM B. If $B$ is an infinite complete Boolean algebra homogeneous in sat, then $B$ is well semifree.

(For precise definitions see §3.)

Although many partial results concerning Theorem A have been achieved, e.g. Monk proved Theorem A under a hypothesis slightly weaker than $\mathrm{GCH}$, we are offering a general approach covering all results obtained so far.

It is not surprising that notions and results about filters and ultrafilters on power set algebras can be translated to ones on complete Boolean algebras. We shall give one such example concerning good ultrafilters. Kunen's proof, see [Ku1, Ch, K], of the existence of good ultrafilters on a power set algebra, can be pushed through in this more general situation as well.

Received by the editors February 10, 1981.

1980 Mathematics Subject Classification. Primary 06E10, 54A25; Secondary 03E05, 06E15, 54G05.

${ }^{1}$ Otherwise $|B| \leqslant|\operatorname{St}(B)| \leqslant 2^{|B|}$. 
At this point we would like to express our thanks to F. D. Tall and P. Simon for stimulating discussions and other help in completing this paper.

2. Corollaries. Let us summarize some well-known consequences of Theorems A and $\mathrm{B}$ with sketches of proofs. In what follows $B$ and $C$ denote infinite complete Boolean algebras, $X$ and $Y$ denote infinite extremally disconnected compact spaces, while $w(X)$ and $w(Y)$ denote their weights.

Corollary 1. $|\operatorname{St}(B)|=2^{|B|}$, or equivalently $|X|=2^{w(X)}$.

Proof. See Corollary 2.

COROLlaRY 2. There are $2^{|B|}$ ultrafilters on $B$ which have character (i.e. the least cardinality of a set of generators) equal to $|B|$.

Proof. Let $\left\{\left\{X_{\alpha}(0), X_{\alpha}(1)\right\}: \alpha<|B|\right\}$ be an independent family of partitions of size 2 on $B$. For any $f:|B| \rightarrow 2$ the system

$$
j_{f}=\left\{X_{\alpha}(f(\alpha)): \alpha<|B|\right\} \cup\left\{-\bigwedge_{\beta \in A} X_{\beta}(f(\beta)): A \in[|B|]^{\omega}\right\}
$$

is centered and thus generates a filter $G_{f}$ on $B$. Let $f \neq g$ be maps from $|B|$ to 2 . Then $G_{f} \neq G_{g}$ and every ultrafilter extending $G_{f}$ is not generated by fewer than $|B|$ elements and is distinct from every ultrafilter extending $G_{g}$ (compare [Ku1]).

Corollaries 1 and 2 solve problems raised by Efimov [E].

Corollary 3. Let $|C| \leqslant|B|$. Then there is a homomorphism $f: B \rightarrow C$ onto $C$, or equivalently if $w(X) \geqslant w(Y)$, then there is an embedding of $Y$ into $X$.

Proof. Let $G$ be a set of free generators of a free subalgebra $F$ of $B$ with $|G|=|B|$. Then every surjection $f: G \rightarrow C$ can be extended to a surjective homomorphism $g: F \rightarrow C$. By injectivity of $C$, there is a homomorphism from $B$ onto $C$ extending $g$.

COROllary 4. There is a continuous surjective map $f: X \rightarrow 2^{w(X)}$.

Proof. This is a topological version of the fact that the complete Boolean algebra $B$, which by Stone duality corresponds to $X$, includes a free subalgebra of size $|B|$. There is a continuous map from $X=\operatorname{St}(B)$ onto $\operatorname{St}(F)=2^{|B|}$. But since $B$ is infinite, $w(X)=w(\operatorname{St}(B))=|B|$.

Corollary 4 solves problem 5 of Ponomarev and Shapiro [P, S].

COROllary 5. The space $X$ includes a copy of itself as a nowhere dense subset (so $X$ is not homogeneous, see [F]).

Proof. It is well known that there is a homeomorphism $f$ from $X$ into $2^{w(X)}$ and onto a nowhere dense $D \subseteq 2^{w(X)}$. By Corollary 4 there is a continuous surjection $g$ : $X \rightarrow 2^{w(X)}$. Let $Y \subseteq X$ be such that $h=g \uparrow Y$ is an irreducible map onto $2^{w(X)}$. Then $Z=h^{-1}(D)$ is nowhere dense in $Y$, and hence in $X$. Since $X$ is extremally disconnected and $h$ is irreducible, $Z$ is homeomorphic to $X$.

Corollary 5 solves problem 4 of $[\mathbf{P}, \mathbf{S}]$. 
COROLlaRY 6. Let $B$ be well semifree. Then there are $2^{|B|}$ good ultrafilters on $B$.

Proof. (Compare $[\mathbf{C h}, \mathbf{K}]$.)

Sublemma 1. Let $(G, \mathcal{F})$ be independent ( for $G \subseteq B, \mathscr{F} \subseteq \operatorname{Part}(B))$ means that

$$
\begin{aligned}
(\forall n<\omega)\left(\forall P_{0}, \ldots, P_{n} \in \mathcal{F}\right)\left(\forall u_{0}\right. & \left.\in P_{0}\right) \cdots\left(\forall u_{n} \in P_{n}\right) \\
& (\forall u \in G)\left(u \wedge u_{0} \wedge \cdots \wedge u_{n} \neq 0_{B}\right) .
\end{aligned}
$$

Let $(G, \mathscr{F})$ be independent. Let $G$ be closed under finite intersections (CUFI). Let $b \in B-\left\{0_{B}\right\}$. Then there is aCUFI $G^{*} \supseteq G,\left|G^{*}\right| \leqslant \max (\omega,|G|)$ and there is an $\mathscr{F}^{*} \subseteq \mathscr{F},\left|\mathscr{F}-\mathscr{F}^{*}\right|<\omega$ so that $\left(G^{*}, \mathscr{F}^{*}\right)$ is independent and either $b \in G^{*}$ or $(-b) \in$ $G^{*}$.

Proof of Sublemma 1. Let $G[X]$ be the closure under finite intersections of $G \cup\{X\}$.

(a) Let $(G[b], \mathscr{F})$ be independent. Then $G^{*}=G[b], \mathscr{F}^{*}=\mathscr{F}$.

(b) Let $(G[b], \mathscr{F})$ be not independent. Let $u \in G, P_{0}, \ldots, P_{n} \in \mathscr{F}, u_{0} \in P_{0}, \ldots, u_{n}$ $\in P_{n}$ witness this fact. Then $G^{*}=G[-b]$ and $\mathscr{F}^{*}=\mathscr{F}-\left\{P_{0}, \ldots, P_{n}\right\}$.

Sublemma 2. Let $\kappa<\operatorname{sat}(B)$. Let $G \subseteq B$ be CUFI. Let $\mathscr{F} \subseteq \operatorname{Part}(B)$. Let $P \in \mathscr{F}$ and $|P|=\kappa$. Let $(G, \mathscr{F})$ be independent. Let $U$ be the filter generated by $G$. Let $f$ : $[\kappa]^{<\omega} \rightarrow U$ be monotonic. Then there is a multiplicative refinement $g$ of $f$ so that $\left(G^{*}, \mathscr{F}^{*}\right)$ is independent where $G^{*}$ is the closure under finite intersections of $G \cup \operatorname{mg}(g)$ and $\mathcal{F}^{*}=\mathscr{F}-\{P\}$.

Proof of Sublemma 2. Let $P=\left\{p_{\alpha}: \alpha<\kappa\right\}$. Let $[\kappa]^{<\omega}=\left\{a_{\alpha}: \alpha<\kappa\right\}$. Define $h_{\alpha}:[\kappa]^{<\omega} \rightarrow B$ by

$$
h_{\alpha}(s)= \begin{cases}f\left(a_{\alpha}\right) \wedge p_{\alpha} & \text { if } s \subseteq a_{\alpha} \\ 0_{B} & \text { otherwise }\end{cases}
$$

Then $h_{\alpha}$ is multiplicative. Define $g:[\kappa]^{<\omega} \rightarrow B$ by $g(s)=V_{\alpha<\kappa} h_{\alpha}(s)$. Then $g(s) \leqslant$ $f(s)$ for each $s$ and $g$ is multiplicative. It is easy to verify that $\left(G^{*}, \mathscr{F}^{*}\right)$ is independent.

Proof of Corollary 6. Since $B$ is well semifree $|B|^{<\operatorname{sat}(B)}=|B|$. So let $B=\left\{b_{\alpha}: \alpha<\nu\right\}$. Let $\delta=\{f:(\exists \kappa<\operatorname{sat}(B))(f$ is a monotonic function with domain $[\kappa]^{<\omega}$ and range $\left.\left.\subseteq B\right)\right\}$. Let $\delta=\left\{f_{\alpha}: \alpha<\nu\right\}$ so that each $f$ is enumerated cofinally many times.

By induction we shall get for each $\alpha<\nu$ and each $\phi: \alpha \rightarrow\{-1,1\}$ a filter $U_{\phi}$ on $B$ generated by a CUFI $G_{\phi}$, and a good family $\mathscr{F}_{\alpha} \subseteq \operatorname{Part}(B)$ so that

(1) $\phi_{1} \subseteq \phi_{2} \rightarrow U_{\phi_{1}} \subseteq U_{\phi_{2}}$,

(2) $\phi_{1}, \phi_{2}$ incompatible $\rightarrow U_{\phi_{1}} \cup U_{\phi_{2}}$ is not centered,

(3) $\operatorname{dom}(\phi)=\alpha \rightarrow\left|G_{\phi}\right| \leqslant \max (|\alpha|, \mu)$ for some $\mu<\operatorname{sat}(B)$,

(4) $\operatorname{dom}(\phi) \leqslant \alpha \rightarrow\left(G_{\phi}, \mathscr{F}_{\alpha}\right)$ is independent,

(5) $\operatorname{dom}(\phi) \geqslant \alpha+1 \rightarrow b_{\alpha} \in G_{\phi}$ or $\left(-b_{\alpha}\right) \in G_{\phi}$, and

(6) if $\operatorname{rng}\left(f_{\alpha}\right) \subseteq U_{\phi} \& \operatorname{dom}(\phi)=\alpha$, then there is a multiplicative refinement $g_{\alpha}$ of $f_{\alpha}$ such that $\operatorname{rng}\left(g_{\alpha}\right) \subseteq U_{\psi}$ for every $\psi \underset{\neq}{\supset} \phi$. 
Since $B$ is well semifree, there is a good independent family $\mathscr{F} \subseteq \operatorname{Part}(B)$ of size $|B|$.

Pick a $P \in \mathscr{F},|P|=\omega$. Let $P=\left\{p_{n}: n<\omega\right\}$. Let $G_{\phi}=\left\{\bigvee_{n \geqslant k} p_{n}: k<\omega\right\}$. Let $U_{\phi}$ be generated by $G_{\phi}$. Let $\mathscr{F}_{0}=\mathscr{F}-\{P\}$.

Use Sublemmas 1 and 2 to get the induction step from $\alpha$ to $\alpha+1$.

Limit stage: Let $\mathscr{F}_{\alpha}=\bigcap_{\beta<\alpha} \mathscr{F}_{\beta}$, for $\phi: \alpha \rightarrow\{-1,1\}$ define $G_{\phi}=\cup_{\beta<\alpha} G_{\phi i \beta}$, $U_{\phi}=\cup_{\beta<\alpha} U_{\phi \uparrow}$.

Now final claim: Let $\chi: \nu \rightarrow\{-1,1\}$. Then $D_{\chi}=\cup_{\alpha<\nu} U_{\chi \uparrow \alpha}$ is a good ultrafilter on $B$. Since $\chi_{1} \neq \chi_{2}$ yields $D_{\chi_{1}} \neq D_{\chi_{2}}$, there are $2^{|B|}=2^{\nu}$ good ultrafilters on $B$.

3. Notation and basic definitions. For a Boolean algebra $B$ and $C \subseteq B$, let $C^{+}=C-\left\{0_{B}\right\}$. If $u \in B^{+}$then $B \uparrow u$ is the partial subalgebra of $B$ with the universe $\{x \in B: x \leqslant u\}$.

(i) $P \subseteq B$ is a partition on $B$ (or a partition of $1_{B}$ ) if it is a maximal disjoint collection of nonzero elements. Part $(B)$ denotes the set of all partitions on $B$.

(ii) $\mathscr{F} \subseteq \operatorname{Part}(B)$ is an independent family of partitions if for any finite subset $\left\{P_{0}, \ldots, P_{n}\right\} \subseteq \mathscr{F}$ and any selector $f \in \Pi_{i \leqslant n} P_{i}, \wedge_{i \leqslant n} f(i) \neq 0_{B}$.

(iii) $C \subseteq B$ is an independent family if for any $x \in C,-x \notin C$ and $\{\{x,-x\}$ : $x \in C\}$ is an independent family of partitions.

(iv) $B$ is semifree if there is an independent family in $B$ of size $|B|$ (see $[\mathbf{K}]$ ).

(v) $\mathscr{F} \subseteq \operatorname{Part}(B)$ is $\kappa$-good (for a cardinal $\kappa \leqslant \operatorname{sat}(B), \kappa>2$ ) if $(\forall \gamma \in \kappa-2$ ) $(|\{P \in \mathscr{F}:|P|=\gamma\}|=|\mathscr{F}|) . \mathscr{F}$ is said to be $\operatorname{good}$ if it is $\operatorname{sat}(B)$-good.

(vi) $B$ is well semifree if there is an independent good family of size $|B|$ of partitions on $B$.

(vii) $D \subseteq B^{+}$is dense in $B$ if $\left(\forall b \in B^{+}\right)(\exists d \in D)(d \leqslant b)$.

(viii) Density of $B, d(B)=\min \{|D|: D$ is dense in $B\}$.

(ix) Saturatedness of $B, \operatorname{sat}(B)=\min \{\kappa \in \operatorname{card}:(\forall P \in \operatorname{Part}(B))(|P|<\kappa)\}$.

(x) $B$ is homogeneous in $d$ if $\left(\forall u \in B^{+}\right)(d(B \backslash u)=d(B))$. $B$ is homogeneous in sat if $\left(\forall u \in B^{+}\right)(\operatorname{sat}(B \uparrow u)=\operatorname{sat}(B))$.

(xi) An ultrafilter $G$ on $B$ is $\kappa$-good (for $\kappa \leqslant \operatorname{sat}(B)$ ) if it is countably incomplete and for every cardinal $\lambda<\kappa$ and every monotonic $f:[\lambda]^{<\omega}=\{X \subseteq \lambda:|X|<\omega\} \rightarrow G$ (i.e. $s \subseteq t \rightarrow f(s) \geqslant f(t))$ there is a multiplicative refinement $g:[\lambda]^{<\omega} \rightarrow G$ of $f$ (i.e. $g(s) \leqslant f(s)$ for each $s$ and $g(s \cup t)=g(s) \wedge g(t))$. $G$ is said to be good if it is sat $(B)$-good.

REMARKS. Since in general $d(B \uparrow u) \leqslant d(B \uparrow v)$ and $\operatorname{sat}(B \uparrow u) \leqslant \operatorname{sat}(B \uparrow v)$ whenever $u \leqslant v$, there is a $P \in \operatorname{Part}(B)$ such that all $B \uparrow u$ 's are homogeneous in $d$ and sat, for all $u \in P$. If $B$ is complete and $P \in \operatorname{Part}(B)$ then $f: B \rightarrow \prod_{u \in P} B \uparrow u$ defined by $f(x)(u)=x \wedge u$ is a bijection and hence $B \cong \prod_{u \in P} B \uparrow u$.

Recall a well-known result of Erdös and Tarski [E, T], that if $B$ is an infinite Boolean algebra, then either $\operatorname{sat}(B)=\kappa^{+}$for some infinite cardinal $\kappa$, or $\operatorname{sat}(B)$ is a weakly inaccessible uncountable cardinal.

There is another definition of a good ultrafilter on a Boolean algebra due to Benda (see [Be]), which gives sufficient and necessary conditions for an ultrafilter so that the corresponding Boolean ultrapower is saturated. Our definition is a formal analogue of Keisler's definition concerning power set algebras (see [Ch, K]). Our 
definition is apparently stronger than Benda's for it implies that even the Boolean valued model of set theory modulo a $\kappa$-good ultrafilter is $\kappa$-saturated. For power set algebras it does not make any difference since any Boolean valued model of set theory modulo an ultrafilter is isomorphic to the Boolean ultrapower.

4. Combinatorial facts. Here we state all facts needed to prove the main theorems. They form five groups: 4.1 contains results enabling the whole business to start, i.e. how to get initial independent families of partitions. 4.2 deals with methods which allow one to enlarge a given independent family. 4.3 is devoted to methods for making partitions in an independent family bigger. In 4.4 it will be shown how to get an independent family in the whole algebra using independent families in partial subalgebras. Finally in 4.5 we shall show that some special types of algebras are well semifree.

In the entire $\S 4, B$ stands for an infinite complete Boolean algebra.

4.1.

Lemma $1[\mathbf{V} ; \mathbf{M}]$. Let $\mathscr{F} \subseteq \operatorname{Part}(B)$. For $P \in \mathscr{F}$, let $P^{\vee}=\{\vee R: R \subseteq P\}$. Let $\mathscr{F}^{\vee} \wedge=\left\{\wedge_{P \in \mathscr{F}} f(P): f \in \Pi_{P \in \mathscr{F}} P^{\vee}\right\}$. If for every $u \in \cup \mathscr{F}$ the set $\{x \leqslant u: x \in$ $\left.\left(\mathscr{F}^{\vee \wedge}\right)^{+}\right\}$is not dense in $B \uparrow u$, then there is a $Q \in \operatorname{Part}(B), Q=\{q(0), q(1)\}$ such that for any $v \in \cup \mathcal{F}, q(0) \wedge v \neq 0_{B}$ and $q(1) \wedge v \neq 0_{B}$.

Proof. Let $u \in \cup \mathscr{F}$. Since $\left\{x \leqslant u: x \in\left(\mathscr{F}^{\vee} \wedge\right)^{+}\right\}$is not dense in $B \uparrow u$ there is an $x(u) \in(B \uparrow u)^{+}$such that for any $z \in\left(\mathscr{F}^{\vee \wedge}\right)^{+}, z \$ x(u)$. Define $y(u)=$ $\wedge_{P \in \mathscr{F}}\left(\vee\left\{v \in P: v \wedge x(u) \neq 0_{B}\right\}\right)$. Then $x(u) \leqslant y(u) \leqslant u$. Pick some $T \subseteq \cup \mathcal{F}$ such that $\{y(u): u \in T\}$ is a maximal pairwise disjoint subsystem of $\{y(u)$ : $u \in \cup \mathscr{F}\}$. Let $q(0)=\bigvee_{u \in T} x(u)$ and $q(1)=1_{B}-q(0)$. Then $Q=\{q(0), q(1)\}$. If $u \in \cup \mathscr{F}$ then by maximality of $T$, there is a $v \in T$ such that $y(u) \wedge y(v) \neq 0_{B}$. So $u \wedge x(v) \neq 0_{B}$ and thus $u \wedge q(0) \neq 0_{B}$. For the same $v, y(u) \wedge(y(v)-x(v)) \neq 0_{B}$, otherwise $x(v) \geqslant y(v) \wedge y(u)$, which is an element of $(\mathscr{F} \vee \wedge)^{+}$, contradicting the definition of $x(v)$. Thus $u \wedge q(1)=u \wedge-q(0) \geqslant u \wedge(y(v)-x(v)) \neq 0_{B}$.

Lemma $2[\mathbf{B}, \mathbf{V}]$. Let $B$ be homogeneous in sat. Let $\nu$ be a cardinal so that $\nu^{+}<\operatorname{sat}(B)$. Then for each family $\{u(\alpha): \alpha<\nu\} \subseteq B^{+}$there is a disjoint refinement, i.e. $\{v(\alpha): \alpha<\nu\} \subseteq B^{+}$such that if $\alpha<\beta<\nu$ then $v(\alpha) \leqslant u(\alpha)$ and $v(\alpha) \wedge v(\beta)=$ $0_{B}$.

Proof. For a detailed proof see $[\mathbf{B}, \mathbf{V}]$. For the sake of completeness here is a sketch of the proof: If $P$ is a family of pairwise disjoint elements of $B$ and $x \in B^{+}$, define $P[x]=\left\{u \in P: u \wedge x \neq 0_{B}\right\}$. If we have a disjoint family $P \subseteq B^{+}$such that $|P[u(\alpha)]| \geqslant \nu$ for every $\alpha<\nu$, we can get a disjoint refinement by a straightforward recursion. The family $P$ we shall also construct by recursion. So suppose $\beta<\nu$, $\cup_{\gamma<\beta} P(\gamma) \subseteq B^{+}$is a disjoint family and for every $\alpha<\nu,\left|\left(\cup_{\gamma<\beta} P(\gamma)\right)[u(\alpha)]\right| \geqslant \nu$ or $=0$. In the first case set $P(\beta)=\varnothing$; otherwise pick a partition $Q$ of $u(\beta)$ such that $|Q|=\nu^{+}$and set $P(\beta)=Q-\cup\{Q[u(\alpha)]: \alpha>\beta \&|Q[u(\alpha)]| \leqslant \nu\}$. Then $|P(\beta)[u(\beta)]|=\nu^{+}$.

LeMma 3. Let $B$ be homogeneous in sat and let $\operatorname{sat}(B)=\kappa$ be weakly inaccessible. Then there is a good independent family of partitions on $B$ that has size $\kappa$. 
Proof. By recursion we shall construct an independent family $\mathscr{F}$ of size $\kappa$ such that $\sup \{|P|: P \in \mathscr{F}\}=\kappa$. It is easy to get a good independent family from $\mathscr{F}$. So suppose $\mathcal{H}$ is an independent family of partitions on $B,|\mathcal{H}|<\kappa$. Given $\tau<\kappa$. Let $T$ be the closure of $\cup \mathcal{H}$ under finite intersection and let $T^{+}=\{u(\alpha): \alpha<\nu\}$ for some $\nu$. Since $\kappa$ is weakly inaccessible and $|\mathcal{H}|<\kappa, \nu^{+}<\kappa$. So by Lemma 2 there is a disjoint refinement $\{v(\alpha): \alpha<\nu\}$ of $T^{+}$. By homogeneity of $B$ in sat, every $v(\alpha)$ can be split into $\tau$ disjoint elements, say $\{v(\alpha, \beta): \beta<\tau\}$. For $\beta>0$ define $x(\beta)=$ $\bigvee_{\alpha<\nu} v(\alpha, \beta)$, and $x(0)=1_{B}-\bigvee_{0<\beta<\tau} x(\beta)$. Then $P=\{x(\beta): \beta<\tau\} \in \operatorname{Part}(B)$ and $\mathcal{H} \cup\{P\}$ is independent, $|P|=\tau$. This procedure allows us to carry out the whole recursion.

4.2.

Definition. Let $\{X(i): i \in I\}$ be a system of sets. A set $\mathcal{S} \subseteq \prod_{i \in I} X(i)$ is called a finitely distinguished family (FDF for short) if for any finite $\mathcal{S}_{0} \subseteq \mathcal{S}$ there is an $i_{0} \in I$ so that $\left|\left\{f\left(i_{0}\right): f \in \mathcal{S}_{0}\right\}\right|=\left|\mathcal{S}_{0}\right|$.

LEMMA 4. If all $X(i)$ 's are infinite, then there is an $F D F \subseteq \subseteq \Pi_{i \in I} X(i)$ of full size, i.e. of power $\left|\prod_{i \in I} X(i)\right|$.

Proof. (a) Let $I$ be finite. Then $\left|\prod_{i \in I} X(i)\right|=|X(j)|$ for some $j \in I$. So $\delta$ can be any family of functions distinct at the $j$ th coordinate.

(b) Let $I$ be infinite, $|I|=\kappa$. We shall proceed by induction over $|I|$. Assume that the assertion holds for all $I$ of size $<\kappa$. Let $\prec$ be a well-ordering of $I$ such that $i \prec j$ implies $|X(i)| \leqslant|X(j)|$. Let $\gamma$ be the order type of $\langle I, \prec\rangle$. Without loss of generality assume $I=\gamma$ and $\prec=\leqslant$. It is obvious that $\kappa \leqslant \gamma<\kappa^{+}$.

Case I. $(\forall \alpha<\gamma)(|\gamma-a|=\kappa)$.

Then there is an injection $\phi:[\gamma]^{<\omega} \rightarrow \gamma$ such that $\phi(s) \geqslant \max (s)$ for each $s \in[\gamma]^{<\omega}$, and there is an injection $\psi_{s}: \prod_{i \in s} X(i) \rightarrow X(\phi(s))$ since $\left|\prod_{i \in s} X(i)\right| \leqslant$ $|X(\phi(s))|$. Define

$$
\chi(h)(i)=\left\{\begin{array}{l}
\psi_{s}(h(s)) \text { if } \phi(s)=i \\
\text { an arbitrary element of } X(i) \text { otherwise }
\end{array}\right.
$$

$\chi$ is an injection from $\prod_{s \in A} \prod_{i \in s} X(i)$ into $\prod_{i \in \gamma} X(i)$, where $A=[\gamma]^{<\omega}-\{\varnothing\}$. Define an injection $\eta: \prod_{i \in \gamma} X(i) \rightarrow \prod_{s \in A} \prod_{i \in s} X(i)$ by $\eta(f)(s)=\langle f(i): i \in s\rangle$. Then $\delta=(\chi \circ \eta)\left(\prod_{i \in \gamma} X(i)\right)$ is an FDF of desired size.

Case II. Case I does not hold.

So $(\exists \alpha<\gamma)(|\gamma-\alpha|<\kappa)$. Let $\alpha_{0}$ be the least such $\alpha$. Clearly $\alpha_{0} \geqslant \kappa$ and $\left|\alpha_{0}-\alpha\right|=\kappa$ for all $\alpha<\alpha_{0}$. By Case I there is an FDF $\mathcal{S}_{0} \subseteq \Pi_{i \in \alpha_{0}} X(i)$ of full size and by the induction assumption an FDF $\mathcal{S}_{1} \subseteq \Pi_{i \in A} X(i)$ of full size (where $A=\gamma-\alpha_{0}$ ). Since either $\left|\prod_{i \in \alpha_{0}} X(i)\right|=\left|\prod_{i \in I} X(i)\right|$ (so $\mathcal{S}_{0}$ can be extended to an FDF $\delta \subseteq \prod_{i \in I} X(i)$ of the same size), or $\left|\prod_{i \in A} X(i)\right|=\left|\prod_{i \in I} X(i)\right|$ (so $\mathcal{S}_{1}$ can be extended to an FDF $\mathcal{S} \subseteq \Pi_{i \in I} X(i)$ of the same size), we are done.

Lemma 5. Let $\mathscr{F}_{1}, \mathscr{F}_{2} \subseteq \operatorname{Part}(B), P \in \operatorname{Part}(B)$. Let $\mathscr{F}_{2}$ be infinite and $\left(\forall Q \in \mathscr{F}_{2}\right)$ $(|Q|=\nu), \nu \geqslant 2$. Let $\mathscr{F}_{1} \cup \mathscr{F}_{2} \cup\{P\}$ be independent. Then there is a family $\mathscr{F}_{2}^{\prime} \subseteq$ $\operatorname{Part}(B)$ such that $\left(\forall R \in \mathscr{F}_{2}^{\prime}\right)(|R|=\nu),\left|\widetilde{F}_{2}^{\prime}\right|=\left|\mathscr{F}_{2}\right|^{|P|}$ and $\mathscr{F}_{1} \cup \mathscr{F}_{2}^{\prime}$ is independent. 
Proof. By Lemma 4 there is an FDF $\delta \subseteq \mathscr{F}_{2}^{P}$ of full size, i.e. $|\delta|=\left|\mathscr{F}_{2}\right|^{|P|}$. Pick $f \in \mathcal{S}, u \in P$. Then $f(u) \in \mathscr{F}_{2}$ and hence $|f(u)|=\nu$. Let $f(u)=\{q(f, u, \alpha): \alpha<\nu\}$. For all $\alpha<\nu$ define $R(f, \alpha)=\bigvee_{u \in P}(q(f, u, \alpha) \wedge u)$.

(i) For $\alpha<\beta<\nu$,

$$
\begin{aligned}
R(f, \alpha) \wedge R(f, \beta) & =\bigvee_{u \in P}(q(f, u, \alpha) \wedge u) \wedge \underset{v \in P}{\vee}(q(f, v, \beta) \wedge v) \\
& =\bigvee_{u, v \in P}(q(f, u, \alpha) \wedge q(f, v, \beta) \wedge u \wedge v)=0_{B}
\end{aligned}
$$

if $u \neq v$, then $u \wedge v=0_{B}$, and if $u=v$, then $q(f, u, \alpha), q(f, v, \beta)$ are two distinct elements of $f(u)$, and hence disjoint.

(ii)

$$
\begin{aligned}
\underset{\alpha<\nu}{\bigvee R(f, \alpha)} & =\underset{\alpha<\nu}{\bigvee} \underset{u \in P}{\bigvee}(q(f, u, \alpha) \wedge u) \\
& =\underset{u \in P}{\bigvee} \underset{\alpha<\nu}{\bigvee}(q(f, u, \alpha) \wedge u)=\underset{u \in P}{\bigvee} u=1_{B}
\end{aligned}
$$

Thus $R(f)=\{R(f, \alpha): \alpha<\nu\}$ is a partition on $B$ of size $\nu$. Let $\mathscr{F}_{2}^{\prime}=\{R(f)$ : $f \in \delta\}$. Then $\mathscr{F}_{2}^{\prime} \subseteq \operatorname{Part}(B)$ and $\left|\mathscr{F}_{2}^{\prime}\right|=\left.\left|\mathscr{F}_{2}\right|\right|^{|P|}$. To show that $\mathscr{F}_{1} \cup \mathscr{F}_{2}^{\prime}$ is independent, pick $n, m<\omega$. Pick $P_{0}, \ldots, P_{n} \in \mathscr{F}_{1}, R\left(f_{0}\right), \ldots, R\left(f_{m}\right) \in \mathscr{F}_{2}^{\prime}$. Pick $v_{0} \in$ $P_{0}, \ldots, v_{n} \in P_{n}, R\left(f_{0}, \alpha_{0}\right) \in R\left(f_{0}\right), \ldots, R\left(f_{m}, \alpha_{m}\right) \in R\left(f_{m}\right)$. Since $\delta$ is an FDF, there is a $u_{0} \in P$ such that $f_{i}\left(u_{0}\right) \neq f_{j}\left(u_{0}\right)$ whenever $i \neq j \leqslant m$. Then

$$
\begin{aligned}
\wedge_{i \leqslant n} v_{i} \wedge \wedge R\left(f_{i}, \alpha_{i}\right) & =\wedge_{i \leqslant m} v_{i} \wedge \wedge \vee_{i \leqslant m}\left(q \in P\left(f_{i}, u, \alpha_{i}\right) \wedge u\right) \\
& \geqslant \wedge_{i \leqslant n} v_{i} \wedge \wedge\left(q\left(f_{i}, u_{0}, \alpha_{i}\right) \wedge u_{0}\right) \\
& =\wedge_{i \leqslant m} v_{i} \wedge \wedge_{i \leqslant m} q\left(f_{i}, u_{0}, \alpha_{i}\right) \wedge u_{0} \neq 0_{B}
\end{aligned}
$$

since (for $i \leqslant n$ ) $v_{i} \in P_{i} \in \mathscr{F}_{1}$, (for $i \leqslant m$ ) $q\left(f_{i}, u_{0}, \alpha_{i}\right) \in f\left(u_{0}\right) \in \mathscr{F}_{2}, u_{0} \in P$ and $\mathscr{F}_{1} \cup \mathscr{F}_{2} \cup\{P\}$ is independent.

4.3.

Lemma 6. Let $\mathscr{F}_{1}, \mathscr{F}_{2} \subseteq \operatorname{Part}(B)$. Let $\left(\forall P \in \mathscr{F}_{2}\right)(|P|=2),\left|\mathscr{F}_{2}\right| \geqslant \omega$, and let $\mathscr{F}_{1} \cup \mathscr{F}_{2}$ be independent. Then there is an $\mathscr{F}_{2}^{\prime} \subseteq \operatorname{Part}(B)$ so that $\left|\mathscr{F}_{2}^{\prime}\right|=\left|\mathscr{F}_{2}\right|$ and $\left(\forall P \in \mathscr{F}_{2}^{\prime}\right)$ $(|P|=\omega)$ and $\mathscr{F}_{1} \cup \mathscr{F}_{2}^{\prime}$ is independent.

Proof. Let $\left|\mathscr{F}_{2}\right|=\nu \geqslant \omega$. Let $\mathscr{F}_{2}=\{P(\alpha): \alpha<\nu\}$. Let $P(\alpha)=\{p(\alpha, 0), p(\alpha, 1)\}$. There is a system of mutually disjoint sets $\left\{A_{\beta}: \beta<\nu\right\}$ such that $\nu=\cup_{\beta<\nu} A_{\beta}$ and $\left|A_{\beta}\right|=\omega$. Let $B_{\beta}$ be the least subalgebra of $B$ containing $\left\{p(\alpha, 0): \alpha \in A_{\beta}\right\}$. Clearly $\operatorname{sat}\left(B_{\beta}\right)=\omega_{1}$. Pick some $R_{\beta} \in \operatorname{Part}\left(B_{\beta}\right)$ of size $\omega$. Pick some $u_{\beta} \in R_{\beta}$. Define $R_{\beta}^{*}=\left(R_{\beta}-\left\{u_{\beta}\right\}\right) \cup\left(u_{\beta} \vee-\vee R_{\beta}\right)$. Then $R_{\beta}^{*} \in \operatorname{Part}(B)$ and has power $\omega$. Finally define $\widetilde{F}_{2}^{\prime}=\left\{R_{\beta}^{*}: \beta<\nu\right\}$. Then $\left|\mathscr{F}_{2}^{\prime}\right|=\nu$ and $\left(\forall P \in \mathscr{F}_{2}^{\prime}\right)(|P|=\omega)$. So it remains to show that $\mathscr{F}_{1} \cup \mathscr{F}_{2}^{\prime}$ is independent.

Pick any $n, m<\omega, Q_{0}, \ldots, Q_{n} \in \mathscr{F}_{1}, u_{0} \in Q_{0}, \ldots, u_{n} \in Q_{n}, \beta_{0}, \ldots, \beta_{m}<\nu, v_{0} \in$ $R_{\beta_{0}}^{*}, \ldots, v_{m} \in R_{\beta_{m}}^{*}$. For each $i \leqslant m$ there is a finite set $D_{i} \subseteq\left\{p(\alpha, 0): \alpha \in A_{\beta_{i}}\right\} \cup$ $\left\{p(\alpha, 1): \alpha \in A_{\beta_{i}}\right\}$ such that $v_{i} \geqslant \wedge D_{i} \neq 0_{B}$, since $\left\{p(\alpha, 0): \alpha \in A_{\beta_{i}}\right\}$ generate $B_{\beta_{i}}$. 
Hence

$$
\bigwedge_{i \leqslant n} u_{i} \wedge \wedge_{i \leqslant m} v_{i} \geqslant \wedge_{i \leqslant n} u_{i} \wedge \wedge \wedge_{i \leqslant m} \wedge D_{i} \neq 0_{B}
$$

for all $D_{i}$ 's are disjoint (as all $A_{\beta}$ 's are disjoint) and elements of $D_{i}$ 's are elements of partitions from $\mathscr{F}_{2}$, and $\mathscr{F}_{1} \cup \mathscr{F}_{2}$ is independent.

Lemma 7. Let $\mathscr{F}_{1}, \mathscr{F}_{2}, \mathscr{F}_{3} \subseteq \operatorname{Part}(B)$. Let $\left|\mathscr{F}_{2}\right|=\omega$ and $\left(\forall P \in \mathscr{F}_{2}\right)(|P|=\tau), \tau \geqslant \omega$. Let $\left(\forall Q \in \mathscr{F}_{3}\right)(|Q|=\omega)$. Let $\mathscr{F}_{1} \cup \mathscr{F}_{2} \cup \mathscr{F}_{3}$ be independent. Then there is an $\mathscr{F}_{2}^{\prime} \subseteq$ $\operatorname{Part}(B)$ of size $\left|\mathscr{F}_{3}\right|$ so that $\left(\forall R \in \mathscr{F}_{2}^{\prime}\right)(|R|=\tau)$ and $\mathscr{F}_{1} \cup \mathscr{F}_{2}^{\prime}$ is independent.

Proof. Let $\mathscr{F}_{2}=\{Q(n): n<\omega\}$ and $Q(n)=\{q(n, \gamma): \gamma<\tau\}$. Let $\mathscr{F}_{3}=\{R(\beta)$ : $\beta<\nu\}$ and $R(\beta)=\{r(\beta, n): n<\omega\}$. Define for every $\beta<\nu, \gamma<\tau, s(\beta, \gamma)=$ $\bigvee_{n<\omega}(q(n, \gamma) \wedge r(\beta, n))$, and $S(\beta)=\{s(\beta, \gamma): \gamma<\tau\}$.

(i) Since $\mathscr{F}_{2} \cup \mathscr{F}_{3}$ is independent, $s(\beta, \gamma) \neq 0_{B}$ for all $\beta<\nu, \gamma<\tau$.

(ii) $s\left(\beta, \gamma_{1}\right) \wedge s\left(\beta, \gamma_{2}\right)=0_{B}$ if $\gamma_{1} \neq \gamma_{2}$. For

$$
\begin{aligned}
s\left(\beta, \gamma_{1}\right) \wedge s\left(\beta, \gamma_{2}\right) & =\underset{n<\omega}{\vee}\left(q\left(n, \gamma_{1}\right) \wedge r(\beta, n)\right) \wedge \underset{m<\omega}{\vee}\left(q\left(m, \gamma_{2}\right) \wedge r(\beta, m)\right) \\
& =\bigvee_{n, m<\omega}\left(q\left(n, \gamma_{1}\right) \wedge r(\beta, n) \wedge q\left(m, \gamma_{2}\right) \wedge r(\beta, m)\right)=0_{B}
\end{aligned}
$$

since if $n=m$ then $q\left(n, \gamma_{1}\right) \wedge q\left(m, \gamma_{2}\right)=0_{B}$, and if $n \neq m$ then $r(\beta, n) \wedge r(\beta, m)$ $=0_{B}$.

(iii)

$$
\begin{aligned}
\bigvee S(\beta) & =\underset{\gamma<\tau}{\bigvee} s(\beta, \gamma)=\underset{\gamma<\tau}{\bigvee} \underset{n<\omega}{\bigvee}(q(n, \gamma) \wedge r(\beta, n)) \\
& =\underset{n<\omega}{\bigvee}(r(\beta, n) \wedge \underset{\gamma<\tau}{\bigvee} q(n, \gamma))=\underset{n<\omega}{\bigvee}\left(r(\beta, n) \wedge 1_{B}\right) \\
& =\underset{n<\omega}{\bigvee} r(\beta, n)=1_{B} .
\end{aligned}
$$

Thus $\mathscr{F}_{2}^{\prime}=\{S(\beta): \beta<\nu\}$ is a family of partitions of size $\nu=\left|\mathscr{F}_{3}\right|$, each partition is of size $\tau$.

To show $\mathscr{F}_{1} \cup \mathscr{F}_{2}^{\prime}$ is independent, pick $n, m<\omega, P_{0}, \ldots, P_{n} \in \mathscr{F}_{1}, v_{0} \in P_{0}, \ldots, v_{n}$ $\in P_{n}, \beta_{0}, \ldots, \beta_{m}<\nu, \gamma_{0}, \ldots, \gamma_{m}<\tau$. We must show that $\wedge_{i \leqslant n} v_{i} \wedge \wedge_{i \leqslant m} s\left(\beta_{i}, \gamma_{i}\right)$ $\neq 0_{B}$.

$$
\begin{aligned}
\bigwedge_{i \leqslant n} v_{i} \wedge \wedge s\left(\beta_{i}, \gamma_{i}\right) & =\wedge_{i \leqslant m} v_{i} \wedge \wedge_{i \leqslant m} \vee\left(q\left(n_{i}, \gamma_{i}\right) \wedge r\left(\beta_{i}, n_{i}\right)\right) \\
& \geqslant \wedge_{i \leqslant n} v_{i} \wedge \wedge_{i \leqslant m}\left(q\left(\bar{n}_{i}, \gamma_{i}\right) \wedge r\left(\beta_{i}, \bar{n}_{i}\right)\right) \neq 0_{B}
\end{aligned}
$$

since $\mathscr{F}_{1} \cup \mathscr{F}_{2} \cup \mathscr{F}_{3}$ is independent (and $\bar{n}_{0}, \ldots, \bar{n}_{m}$ are chosen pairwise distinct).

4.4 .

Lemma 8. Let $P \in \operatorname{Part}(B)$. Let there be for every $u \in P$ an infinite $\kappa$-good independent family $\mathscr{F}_{u}$ of partitions on $B \nmid u, \kappa>2$. Then there is a $\kappa$-good independent family $\mathscr{F} \subseteq \operatorname{Part}(B)$ of size $\prod_{u \in P}\left|\mathscr{F}_{u}\right|$. 
Proof. Let $\mathscr{F}_{u}=\cup_{2 \leqslant \gamma<\kappa} \mathscr{F}_{u, \gamma}$ for every $u \in P$, and $\left|\mathscr{F}_{u}\right|=\left|\mathscr{F}_{u, \gamma}\right|=\nu_{u}$. Let $\mathscr{F}_{u, \gamma}=$ $\left\{P_{u, \gamma}(\alpha): \alpha<\nu_{u}\right\}$ and $P_{u, \gamma}(\alpha)=\left\{p_{u, \gamma}(\alpha, \beta): \beta<\gamma\right\}$. Pick an FDF $\delta \subseteq \Pi_{u \in P} \nu_{u}$ of full size (by Lemma 4). Pick an $f \in \mathcal{S}$. For $\beta<\gamma, 2 \leqslant \gamma<\kappa$, define $P_{\gamma}(f, \beta)=$ $\bigvee_{u \in P} p_{u, \gamma}(f(u), \beta)$. Let $P_{\gamma}(f)=\left\{P_{\gamma}(f, \beta): \beta<\gamma\right\}$ and $\mathscr{F}_{\gamma}=\left\{P_{\gamma}(f): f \in \delta\right\}$. Finally let $\mathscr{F}=\bigcup_{2 \leqslant \gamma<\kappa} \mathscr{F}_{\gamma}$.

(i) If $\beta_{1} \neq \beta_{2}$ then $P_{\gamma}\left(f, \beta_{1}\right) \wedge P_{\gamma}\left(f, \beta_{2}\right)=0_{B}$. For

$$
\begin{aligned}
P_{\gamma}\left(f, \beta_{1}\right) \wedge P_{\gamma}\left(f, \beta_{2}\right) & =\bigvee_{u \in P} p_{u, \gamma}\left(f(u), \beta_{1}\right) \wedge \vee_{v \in P} p_{v, \gamma}\left(f(v), \beta_{2}\right) \\
& =\bigvee_{u, v \in P}\left(p_{u, \gamma}\left(f(u), \beta_{1}\right) \wedge p_{v, \gamma}\left(f(v), \beta_{2}\right)\right)=0_{B}
\end{aligned}
$$

since if $u=v$ then $p_{u, \gamma}\left(f(u), \beta_{1}\right) \wedge p_{v, \gamma}\left(f(v), \beta_{2}\right)=0_{B}$ as they are distinct elements of $P_{u, \gamma}(f(u))$, if $u \neq v$ then $p_{u, \gamma}\left(f(u), \beta_{1}\right) \leqslant u$ and $p_{v, \gamma}\left(f(v), \beta_{2}\right) \leqslant v$ and $u \wedge v=0_{B}$.

(ii) $\vee P_{\gamma}(f)=1_{B}$. For

$$
\bigvee P_{\gamma}(f)=\underset{\beta<\gamma}{\bigvee} P_{\gamma}(f, \beta)=\underset{\beta<\gamma}{\bigvee} \underset{u \in P}{\bigvee} P_{u, \gamma}(f(u), \beta)=\underset{u \in P}{\bigvee} u=1_{B} .
$$

Therefore $\mathscr{F}_{\gamma}$ is a family of partitions of size $\gamma$ on $B,\left|\mathscr{F}_{\gamma}\right|=|\mathscr{F}|=|\delta|$. So $\mathscr{F}$ is a $\kappa$-good family. We shall show that $\mathscr{F}$ is independent.

Pick $n, m<\omega$. Pick $\gamma_{0}, \ldots, \gamma_{n}<\kappa, f_{0}, \ldots, f_{m} \in \mathcal{S}, \beta_{0}<\gamma_{0}, \ldots, \beta_{n}<\gamma_{n}$. We shall show that $\wedge_{i \leqslant n} \wedge_{j \leqslant m} P_{\gamma_{i}}\left(f_{j}, \beta_{i}\right) \neq 0_{B}$. Pick $u \in P$ so that all $f_{j}(u)$ 's are distinct (possible since $f \in \mathcal{S}$ and $\mathcal{S}$ is an FDF). Then

$$
\bigwedge_{i \leqslant n} \wedge P_{\gamma_{i}}\left(f_{j}, \beta_{i}\right) \geqslant \bigwedge_{i \leqslant n} \bigwedge_{j \leqslant m} p_{u, \gamma_{i}}\left(f_{j}, \beta_{i}\right) \neq 0_{B}
$$

since $\mathscr{F}_{u}$ is independent.

4.5.

LemMa 9 [E, K]. Let $B$ be atomic. Then $B$ is well semifree.

Proof. Let $\operatorname{sat}(B)=\kappa^{+} \geqslant \omega_{1}$. Then $B \cong \mathscr{P}(\kappa)$. Since every set of size $\kappa$ can be split into $\kappa$ disjoint sets of size $\kappa$, it is easy to get (by induction) a countable independent family of partitions on $B$, each of size $\kappa$. Denote this family $\mathscr{F}$. Then there is a $P \in \mathscr{F}_{F}, \mathscr{F}_{2} \subseteq \mathscr{F}$ so that $\mathscr{F}=\{P\} \cup \mathscr{F}_{2},|P|=\kappa$ and $\left|\mathscr{F}_{2}\right|=\omega$. By Lemma 5 there is a family $\mathscr{F}_{2}^{\prime} \subseteq \operatorname{Part}(B)$ of size $\left|\mathscr{F}_{2}\right|^{|P|}=\omega^{\kappa}=2^{\kappa}=|B|$ such that $\left(\forall R \in \mathscr{F}_{2}^{\prime}\right)$ $(|R|=\kappa)$ and $\mathscr{F}_{2}^{\prime}$ is independent. Now it is easy to get a $\kappa$-good independent family from $\mathscr{F}_{2}^{\prime}$.

LEMma 10. Let $B$ be homogeneous in sat and $d$. Let $\operatorname{sat}(B)=\kappa$ be weakly inaccessible. Then $B$ is well semifree.

Proof. Let $d(B)=\lambda$. Then $\omega \leqslant \kappa \leqslant \lambda$. Notice that $|B|=\lambda^{<\kappa}=\sup \left\{\lambda^{\gamma}: \gamma<\kappa\right\}$. For pick a dense subset $D \subseteq B$ of size $\lambda$. Since every element of $B$ can be expressed as a sum of fewer than $\kappa$ elements from $D,|B| \leqslant \lambda^{<\kappa}$. If $\tau<\kappa$, there is a $P \in \operatorname{Part}(B)$ of size $\tau$. Since $B$ is complete, $B \cong \prod_{u \in P} B \uparrow u$. By homogeneity of $B$ in $d$, for each $u \in P,|B| u \mid \geqslant d(B \nmid u)=d(B)=\lambda$. Hence $|B| \geqslant \lambda^{\tau}$. Since this is true for all $\tau<\kappa,|B| \geqslant \lambda^{<\kappa}$. 
Now let $\mu$ be the least cardinal so that $\lambda^{<\kappa}=\mu^{<\kappa}$. Then if $\tau<\mu, \tau^{<\kappa}<\lambda$ (otherwise $\tau^{<\kappa}=\left(\tau^{<\kappa}\right)^{<\kappa} \geqslant \lambda^{<\kappa}$ ) and $\mu=2$ or $\mu>2^{<\kappa}$.

If $\mu=2$, then by Lemma 3 there is a good independent family $\mathscr{F} \subseteq \operatorname{Part}(B)$ of size $\kappa \geqslant \omega$. If $\mu>2^{<\kappa}$ then a good independent family of size $\kappa$ given by Lemma 3 can be extended by recursion to a good independent family $\mathscr{F} \subseteq \operatorname{Part}(B)$ of size $\mu$.

Sketch of the recursion: Suppose we have $\mathcal{H}=\{P(\alpha): \alpha \subset \tau\}$, an independent family so that $\sup \{|P(\alpha)|: \alpha<\tau\}=\kappa$ and $\kappa \leqslant \tau<\mu$. Then $\left|P(\alpha)^{\vee}\right| \leqslant 2^{<\kappa}$ since $|P(\alpha)|<\kappa$. Thus $\left|\cup_{\alpha<\tau} P(\alpha)^{\vee}\right|=\nu<\mu$. Therefore $\left|\mathcal{H}^{\vee \wedge}\right|=\nu^{<\kappa}<\lambda=d(B)=$ $d(B \uparrow u)$ for every $u \in \cup \mathcal{H}$. So all assumptions of Lemma 1 are fulfilled and hence there is a $P(\tau) \in \operatorname{Part}(B)$ so that $\{P(\alpha): \alpha \leqslant \tau\}$ is independent. ${ }^{2}$

Thus in both cases $\left(\mu=2\right.$ or $\left.\mu>2^{<\kappa}\right)$ we have a good independent family $\mathscr{F} \subseteq \operatorname{Part}(B)$ of size $\max (\mu, \kappa)$. Let $\mathscr{F}=\cup_{2 \leqslant \gamma<\kappa} \mathscr{F}_{\gamma}$ and $\left|\mathscr{F}_{\gamma}\right|=|\mathscr{F}|=\max (\mu, \kappa)$ and $\left(\forall P \in \mathscr{F}_{\gamma}\right)(|P|=\gamma)$. By recursion using Lemma 5, every $\mathscr{F}_{\gamma}$ can be replaced by $\mathscr{F}_{\gamma}^{\prime}$ of size $\max (\mu, \kappa)^{<\kappa}$ such that $\mathscr{F}^{\prime}=\cup_{2 \leqslant \gamma<\kappa} \mathscr{F}_{\gamma}^{\prime}$ is independent and good. The size of $\mathscr{F}^{\prime}$ is $\kappa^{<\kappa} \geqslant 2^{<\kappa}=\mu^{<\kappa}=\lambda^{<\kappa}=|B|$ (if $\mu=2$ ) or $\mu^{<\kappa}=\lambda^{<\kappa}=|B|$ (if $\mu>2^{<\kappa}$ ), so $\left|\mathscr{F}^{\prime}\right|=|B|$. Thus $B$ is well semifree.

Lemma 11. Let $B$ be homogeneous in sat and $d$. Let $\operatorname{sat}(B)=\kappa^{+}$. Then $B$ is well semifree.

Proof. Let $d(B)=\lambda$. Notice that $|B|=\lambda^{\kappa}$ (see proof of Lemma 10). Let $\mu$ be the least cardinal such that $\mu^{\kappa}=\lambda^{\kappa}$. Then if $\tau<\mu, \tau^{\kappa}<\lambda$ and $\mu=2$ or $\mu>2^{\kappa}$.

One can easily obtain an independent countable family of partitions on $B$, each of size $\kappa$, denote it $\mathscr{F}_{1}$. If $\mu>2^{<\kappa}$, then as in the previous proof we can extend (by recursion) $\mathscr{F}_{1}$ by a family $\mathscr{F}_{2}$ of size $\mu$ such that $\left(\forall P \in \mathscr{F}_{2}\right)(|P|=2)$. By Lemma 6 there is a family $\mathscr{F}_{2}^{\prime} \subseteq \operatorname{Part}(B),\left|\mathscr{F}_{2}^{\prime}\right|=\left|\mathscr{F}_{2}\right|=\mu$ such that $\left(\forall P \in \mathscr{F}_{2}^{\prime}\right)(|P|=\omega)$ and $\mathscr{F}_{1} \cup \mathscr{F}_{2}^{\prime}$ is independent. By Lemma 7 there is a family $\mathscr{F}_{1}^{\prime} \subseteq \operatorname{Part}(B)$ of size $\left|\mathscr{F}_{2}^{\prime}\right|$ such that $\left(\forall P \in \mathscr{F}_{1}^{\prime}\right)(|P|=\kappa)$ and $\mathscr{F}_{1}^{\prime}$ is independent. Thus in every case we have a good independent family $\mathscr{F} \subseteq \operatorname{Part}(B)$ of size $\max (\mu, \kappa)$. Hence $\mathscr{F}=\cup_{2 \leqslant \gamma \leqslant \kappa} \mathscr{F}_{\gamma}$ and $|\mathscr{F}|=\left|\mathscr{F}_{\gamma}\right|=\max (\mu, \kappa)$, and $\left(\forall P \in \mathscr{F}_{\gamma}\right)(|P|=\gamma)$. Now, by recursion using Lemma 5, each $\mathscr{F}_{\gamma}$ can be replaced by a family $\mathscr{F}_{\gamma}^{\prime}$ of size $\max (\mu, \kappa)^{\kappa}$ such that $\mathscr{F}^{\prime}=\bigcup_{2 \leqslant \gamma \leqslant \kappa} \mathscr{F}_{\gamma}^{\prime}$ is independent and hence good and of size $\max (\mu, \kappa)^{\kappa}=|B|$. So $B$ is well semifree.

\section{Main theorems.}

Theorem A. Let $B$ be an infinite complete Boolean algebra. Then $B$ is semifree.

Proof. Let $A$ be the set of all atoms of $B$. Let $u=\vee A, v=-u$. Then $B \cong B \uparrow u$ $\times B \uparrow v$.

If $|B|=|B \nmid u|$, we are done, since $B \uparrow u$ is atomic and by Lemma 9 there is a good independent family in $B \uparrow u$ of full size, i.e. of size $|B|$. One can easily obtain a good independent family of size $|B|$ in $B$.

So assume that $|B|>|B| u \mid$. Then $|B|=|B| v \mid$. Denote $B \uparrow v$ by $C$. Since $C$ is atomless, there is a $P \in \operatorname{Part}(C)$ so that for every $w \in P, C \uparrow w$ is homogeneous in

${ }^{2}$ Using Lemmas 6 and 7 we obtain a good independent family of size $\mu$. 
sat and $d . C \cong \prod_{w \in P} C \uparrow w$ for $C$ is complete. By Lemmas 10 and 11 every $C \uparrow w$ is well semifree, hence semifree. By Lemma $8 C$ is semifree, since all independent families in $C$, $w$ 's generate an independent family in $C$ of size $\prod_{w \in P}|C| w|=| C \mid$ since $C$ is complete. Thus $B$ is semifree, too.

THEOREM B. Let $B$ be an infinite complete Boolean algebra homogeneous in sat. Then $B$ is well semifree.

Proof. Since $B$ is homogeneous in sat, $B$ is atomless. Thus there is a $P \in \operatorname{Part}(B)$ such that for each $u \in P, B \uparrow u$ is homogeneous in $d$ (and in sat, also). By Lemmas 10 and 11 all $B \uparrow u$ 's are well semifree. Let $\mathscr{F}_{u}$ witness well-semifreeness of $B \uparrow u$. By Lemma 8 there is a good independent family $\mathscr{F}$ in $B$ of size

$$
\prod_{u \in P}\left|\mathscr{F}_{u}\right|=\prod_{u \in P}|B \uparrow u|=\left|\prod_{u \in P} B \uparrow u\right|=|B|
$$

since $B$ is complete. Hence $B$ is well semifree.

\section{REFERENCES}

[Be] M. Benda, Reduced products, filters and Boolean ultrapowers, Ph.D. Thesis, University of Wiscon$\sin , 1970$.

[B, V] B. Balcar and P. Vojtáš, Refining systems on Boolean algebras, Lecture Notes in Math., vol. 619, Springer-Verlag, Berlin and New York, 1977, pp. 45-68.

[Bla] A. Blaszczyk, On mappings of extremally disconnected compact spaces onto Cantor cubes, Proc. Colloq. Topology, Budapest, 1978.

[Bu] L. Bukovsky, Boolean ultrapowers and elementary equivalence, Abstract of 4th ICLMPS Bucharest, 1971, pp. 13-14.

$[\mathrm{C}, \mathrm{H}] \mathrm{W}$. W. Comfort and A. W. Hager, Cardinality of $\tau$-complete Boolean algebras, Pacific J. Math. 40 (1972), 541-545.

[C, N] W. W. Comfort and S. Negrepontis, The theory of ultrafilters, Springer-Verlag, Berlin and New York, 1974.

[Ch, K] C. C. Chang and H. J. Keisler, Model theory, North-Holland, Amsterdam, 1973.

[E] B. A. Efimov, Extremally disconnected compact spaces and absolutes, Trudy Moskov. Mat. Obšč. 23 (1970), 235-276. (Russian)

[E, K] R. Engelking and M. Karlowicz, Some theorems of set theory and their topological consequences, Fund. Math. 57 (1965), 275-285.

[E, T] P. Erdös and A. Tarski, On families of mutually exclusive sets, Ann. of Math. (2) 44 (1943). 315-329.

[F] Z. Frolik, Fixed points of maps of extremally disconnected spaces and complete Boolean algebras, Bull. Acad. Polon. Sci. Sér. Sci. Math. 16 (1968), 269-275.

[H] F. Hausdorff, Uber zwei Sätze von G. Fichtenholz und L. Kantorovich, Studia Math. 6 (1936), 18-19.

[J] T. Jech, Set theory, Academic Press, New York, 1978.

[K] S. Kesl'yakov, Free subalgebras of complete Boolean algebras and spaces of continuous functions, Sibirsk. Mat. Ž. 14 (1973), 569-581.

[K1] H. J. Keisler, Good ideals in fields of sets, Ann. of Math. (2) 79 (1964), 338-359.

[K2] __ Ultraproducts and saturated models, Indag. Math. 26 (1964), 178-1868.

[Ke] J. Ketonen, Everything you wanted to know about ultrafilters but were afraid to ask, Ph.D. Thesis, University of Wisconsin, 1971.

[Ko] S. Koppelberg, Free subalgebras of complete Boolean algebras, Notices Amer. Math. Soc. 20 (1973), A-418.

[Kul] K. Kunen, Ultrafilters and independent sets, Trans. Amer. Math. Soc. 172 (1972), 291-306.

[Ku2] _ Weak p-points in $\beta \mathbf{N}-\mathbf{N}$, Colloq. Math. Soc. (J. Bolyai, ed.), no. 23, Topology, Budapest, 1980, pp. 741-749.

[Ma] D. C. Makinson, On the number of ultrafilters on infinite Boolean algebras, Z. Math. Logik Grundlag. Math. 15 (1969), 121-122. 
[Mn] R. Mansfield, The theory of Boolean ultrapowers, Ann. Math. Logic 2 (1971), 292-323.

[M] J. D. Monk, On free subalgebras of complete Boolean algebras, Arch. Math. (Basil) 29 (1977), 113-116.

[P, S] V. I. Ponomarev and L. B. Shapiro, Absolutes of topological spaces and their continuous maps, Russian Math. Surveys 31 (1976), no. 5, 138-154.

[P1] B. Pospišil, Remark on bicompact spaces, Ann. of Math. (2) 30 (1937), 845-846.

[P2] _ On bicompact spaces, Publ. Fac. Sci. Univ. Masaryk 270 (1939), 3-16.

[V] D. Vladimirov, Boolean algebras, “Nauka”, Moskow, 1969.

ČKd Praha, O. P. Závod Polovodiče, 14000 Praha, Czechoslovakia

University of Toronto, Department of Mathematics, Toronto, Ontario M5S 1A1, Canada 